\title{
Gradiente $\mathrm{SpO}_{2}-\mathrm{SaO}_{2}$ Durante Ventilação Mecânica em Anestesia e Terapia Intensiva * $\mathrm{SpO}_{2}-\mathrm{SaO}_{2} \mathrm{Gap}$ During Mechanical Ventilation in Anesthesia and Intensive Care
}

Pablo Escovedo Helayel ${ }^{1}$, Getúlio R. de Oliveira Filho, TSA ${ }^{2}$, Lúcia Marcon ${ }^{3}$, Flávio Hülse Pederneiras ${ }^{4}$, Marcos Antônio Nicolodi, TSA ${ }^{5}$, Sérgio Galluf Pederneiras, TSA ${ }^{6}$

\section{RESUMO}

Helayel PE, Oliveira Filho GR, Marcon L, Pederneiras FH, Nicolodi MA, Pederneiras SG - Gradiente $\mathrm{SpO}_{2}-\mathrm{SaO}_{2}$ Durante Ventilação Mecânica em Anestesia e Terapia Intensiva

Justificativas e Objetivos - A saturação periférica da oxihemoglobina $\left(\mathrm{SpO}_{2}\right)$ é freqüentemente utilizada para guiar alterações do regime ventilatório. Valores de $\mathrm{SpO}_{2}$ iguais ou superiores a $96 \%$ são necessários para garantir saturação arterial da oxihemoglobina $\left(\mathrm{SaO}_{2}\right)$ superiores a $90 \%$, em pacientes de terapia intensiva. Este estudo teve por objetivo determinar concordância entre valores de $\mathrm{SpO}_{2}$ e $\mathrm{SaO}_{2}$ e delimitar a menor $\mathrm{SpO}_{2}$ associada a valores de $\mathrm{SaO}_{2}$ iguais ou superiores a $90 \%$.

Método - Foram incluídos prospectivamente 120 pacientes adultos, de ambos os sexos, submetidos à anestesia geral com ventilação mecânica ou em tratamento intensivo. Amostras de sangue arterial foram coletadas por punção arterial ou por aspiração de linha arterial, utilizando técnica anaeróbia, em seringas heparinizadas. As amostras foram analisadas imediatamente após a coleta. A $\mathrm{SpO}_{2}$ foi medida por oxímetro de pulso durante a coleta, no indicador da mão contra-lateral, utilizando a onda dicrótica de pulso arterial como parâmetro de adequação.

Resultados - Foram analisadas 228 amostras. A diferença entre os valores de $\mathrm{SpO}_{2}$ e $\mathrm{SaO}_{2}$ variou entre $-7,10 \%$ e $15,20 \%$, sendo a diferença média igual a $-0,20 \% \pm 2,02 \%$. SpO $-\mathrm{SaO}_{2}$ maiores que $4,04 \%$ (dois desvios padrão da diferença) ocorreram em $4,72 \%$ das amostras. A determinação gráfica da $\mathrm{SpO}_{2}$, mínima para garantir a $\mathrm{SaO}_{2}$ acima de $90 \%$, mostrou que somente valores de $\mathrm{SpO}_{2}$ iguais ou superiores a $99 \%$ não se associaram à ocorrência de nenhum valor de $\mathrm{SaO}_{2}$ inferior a $90 \%$.

Conclusões - Embora a diferença entre os valores de $\mathrm{SpO}_{2}$ e $\mathrm{SaO}_{2}$ tenham se localizado entre mais e menos $5 \%$ em $97 \%$ dos

\footnotetext{
* Recebido do (Received from) Hospital Governador Celso Ramos CET/SBA Integrado de Anestesiologia da SES-SC, Florianópolis, SC

1. $M E_{2}$ do CET/SBA da SES-SC

2. Responsável pelo CET/SBA da SES-SC

3. Enfermeira da Unidade de Tratamento Intensivo

4. $M E_{1}$ do CET/SBA da SES-SC

5. Anestesiologista do Hospital Governador Celso Ramos

6. Co-Responsável do CET/SBA da SES-SC
}

Apresentado (Submitted) em 01 de dezembro de 2000

Aceito (Accepted) para publicação em 09 de janeiro de 2001

Correspondência para (Mail to):

Dr. Getúlio Rodrigues. de Oliveira Filho

Rua José Cândido Silva 179/402

88075-250 - Florianópolis, SC

E-mail:grof@th.com.br

(C) Sociedade Brasileira de Anestesiologia, 2001 pares analisados, somente valores acima de $99 \%$ excluíram pacientes com $\mathrm{SaO}_{2}$ menor que $90 \%$.

UNITERMOS: MONITORIZAÇÃO: oximetria de pulso; VENTILAÇÃO: ventilação mecânica

\section{SUMMARY}

Helayel PE, Oliveira Filho GR, Marcon L, Pederneiras FH, Nicolodi MA, Pederneiras SG - $\mathrm{SpO}_{2}-\mathrm{SaO}_{2}$ Gap During Mechanical Ventilation in Anesthesia and Intensive Care

Background and Objectives - Peripheral oxy-hemoglobin saturation $\left(\mathrm{SpO}_{2}\right)$ is commonly used to guide ventilator settings. $\mathrm{SpO}_{2}$ values equal to, or higher than $96 \%$ are necessary to assure arterial oxy-hemoglobin saturation $\left(\mathrm{SaO}_{2}\right)$ higher than $90 \%$ in intensive care patients. This study aimed to determine $\mathrm{SpO}_{2}-\mathrm{SaO}_{2}$ gap and to establish the lowest $\mathrm{SpO}_{2}$ associated to $\mathrm{SaO}_{2}$ values equal to, or higher than $90 \%$.

Methods - Participated in this prospective study 120 adult patients of both genders submitted to general anesthesia and mechanical ventilation or under intensive care. Arterial blood samples were obtained by arterial puncture or central line aspiration using the anaerobic technique and heparin-containing syringes. Samples were analyzed immediately after collection. $\mathrm{SpO}_{2}$ was measured during collection with the pulse oximeter placed on the opposite second finger and using arterial pulse dichroic wave as the adequacy parameter.

Results - Two hundred and twenty-eight samples were analyzed. The difference between $\mathrm{SpO}_{2}$ and $\mathrm{SaO}_{2}$ ranged between $-7.10 \%$ and $15.2 \%$, being $-0.20 \% \pm 2.02 \%$ the mean difference (bias). $\mathrm{SpO}_{2}-\mathrm{SaO}_{2}$ higher than $4.04 \%$ (two standard deviations of the difference) was observed in $4.72 \%$ of samples. Graphical analysis of the lowest $\mathrm{SpO}_{2}$ to assure an $\mathrm{SaO}_{2}$ higher than $90 \%$ has shown that only $\mathrm{SpO}_{2}$ values equal to, or higher than $99 \%$ were not associated to $\mathrm{SaO}_{2}$ values below $90 \%$.

Conclusions - Although $\mathrm{SpO}_{2}-\mathrm{SaO}_{2}$ gap remaining between plus or minus $5 \%$ for $97 \%$ of the samples, only $\mathrm{SpO}_{2}$ values higher than $99 \%$ excluded patients with $\mathrm{SaO}_{2}$ values below $90 \%$.

KEY WORDS - MONITORING: pulse oximetry; VENTILATION: mechanical ventilation

\section{INTRODUÇÃO}

A saturação periférica da oxihemoglobina é uma estimatiAva de sua saturação no sangue arterial amplamente utilizada na monitorização de pacientes durante anestesia e em terapia intensiva. Durante ventilação mecânica, a $\mathrm{SpO}_{2}$ tem sido utilizada para determinar a adequação de mudanças feitas no regime ventilatório, como alterações da fração inspirada de oxigênio, valores de pressão positiva tele-expiratória e desmame. Os valores de $\mathrm{SpO}_{2}$ tradicionalmente 
associados com $\mathrm{SaO}_{2}$ acima de $90 \%$, ou seja, normoxemia, variam entre 92 e $94 \%{ }^{1}$. Entretanto, recentemente, a ausência de hipoxemia só foi verificada quando os valores da $\mathrm{SpO}_{2}$ foram iguais ou maiores que $96 \%{ }^{2}$. Estes achados possuem implicações clínicas óbvias.

Este estudo teve como objetivos estudar os limites de concordância entre os valores de $\mathrm{SpO}_{2}$ e $\mathrm{SaO}_{2}$ em pacientes anestesiados e criticamente enfermos durante ventilação mecânica e estabelecer os valores mínimos de $\mathrm{SpO}_{2}$ associados a saturações arteriais da oxihemoglobina superiores a $90 \%$.

\section{MÉTODO}

Com a aprovação da Comissão de Ética Médica do Hospital Governador Celso Ramos, foram incluídos prospectivamente 120 pacientes adultos, de ambos os sexos, submetidos à ventilação mecânica durante anestesia geral ou terapia intensiva, com fração inspirada de oxigênio entre $40 \%$ e $100 \%$, independentemente dos demais parâmetros de ventilação utilizados ou do estado hemodinâmico. Foram excluídos os pacientes em circulação extracorpórea, os ictéricos, os que tinham esmalte nas unhas e os anêmicos (hemoglobina menor que $9 \mathrm{~g} \cdot \mathrm{ml}^{-1}$ ). Também não foram incluídos pacientes cujas gasometrias apresentaram $\mathrm{SaO}_{2}$ igual ou menor que $75 \%$, por possibilidade de contaminação venosa da amostra durante a punção arterial. Amostras de sangue arterial foram coletadas por punção da artéria radial ou por aspiração de linha arterial, utilizando técnica anaeróbia, em seringas heparinizadas. As amostras foram analisadas imediatamente após a coleta. Durante a coleta, foram anotados os valores de $\mathrm{SpO}_{2}$, a partir de oxímetro de pulso com sensores reutilizáveis digitais, utilizando a onda dicrótica de pulso arterial como parâmetro de adequação da medida.

Os valores de $\mathrm{SpO}_{2}$ e de $\mathrm{SaO}_{2}$ foram comparados pelo teste U de Mann-Whitney e sua concordância foi avaliada pelo método de Bland-Altman. Para determinar o menor valor de $\mathrm{SpO}_{2}$ associado com $\mathrm{SaO}_{2}$ igual ou superior a $90 \%$, foi utilizado um gráfico $\mathrm{SaO}_{2}, \mathrm{SpO}_{2}$ no qual foram desenhadas duas linhas. A primeira linha, vertical, definiu o limite de $\mathrm{SaO}_{2}$ em $90 \%$ e a segunda, horizontal, cruzou o eixo das ordenadas no valor de $\mathrm{SpO}_{2}$ acima do qual não foram observados valores de $\mathrm{SaO}_{2}$ menor que de $90 \%$.

\section{RESULTADOS}

Foram analisadas 228 amostras de 120 pacientes cujos dados demográficos estão representados na tabela I. Os valores de $\mathrm{SpO}_{2}$ diferiram significativamente dos da $\mathrm{SaO}_{2}$ (Tabela II). A diferença entre os valores de $\mathrm{SpO}_{2}$ e $\mathrm{SaO}_{2}$ variou entre $-7.10 \%$ e $15,20 \%$, sendo a diferença média igual a - 0,20\%, com desvio padrão igual a 2,02\%. O gradiente $\mathrm{SpO}_{2}-\mathrm{SaO}_{2}$ localizou-se entre menos e mais $4,04 \%$ (dois desvios padrão da diferença média) em $95,28 \%$ das amostras (Figura 1).
Tabela I - Dados Demográficos

\begin{tabular}{lc}
\hline Idade (anos) * & $52,94 \pm 16,56$ \\
Sexo \# & \\
$\quad$ Masculino & 66 \\
$\quad$ Feminino & 54 \\
Peso $(\mathrm{kg})^{*}$ & $71,60 \pm 15,97$ \\
Altura $(\mathrm{m})^{*}$ & $1,65 \pm 0,09$ \\
\hline
\end{tabular}

* Valores expressos pela Média \pm DP

\# Número de casos

Tabela II - Comparação entre os Valores de $\mathrm{SaO}_{2}$ e $\mathrm{SpO}_{2}$

\begin{tabular}{lc}
\hline Parâmetro & $\begin{array}{c}\text { Mediana [quartil inf.; superior] } \\
\text { (Mínimo - Máximo) }\end{array}$ \\
\hline $\mathrm{SaO}_{2}$ & $99,8[98,5 ; 100]{ }^{*}$ \\
& $83-100$ \\
$\mathrm{SpO}_{2}$ & $99[98 ; 100]$ \\
& $84-100$
\end{tabular}

${ }^{*} \mathrm{p}<0,05$ comparado à $\mathrm{SpO}_{2}$

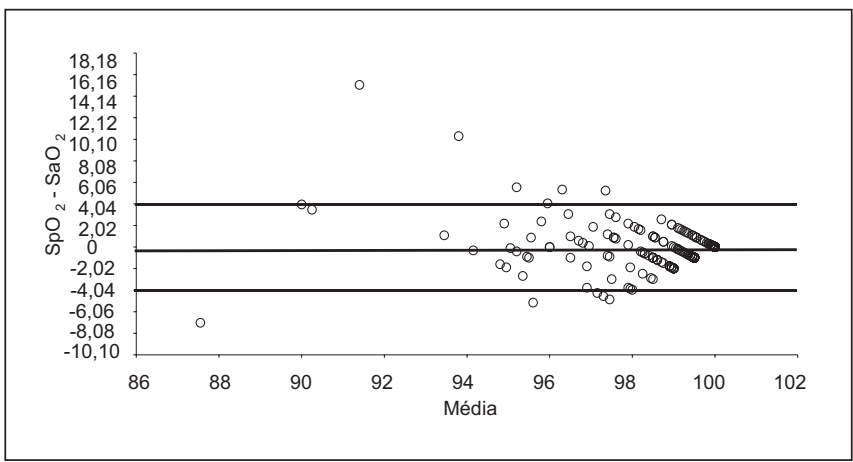

Figura 1 - Diagrama do Bland-Altman para a $\mathrm{SpO}_{2}-\mathrm{SaO}_{2}$

A determinação gráfica da $\mathrm{SpO}_{2}$ mínima para garantir a $\mathrm{SaO}_{2}$ acima de $90 \%$ mostrou que somente valores de $\mathrm{SpO}_{2}$ iguais ou superiores a 99\% não se associaram à ocorrência de nenhum valor de $\mathrm{SaO}_{2}$ inferior a $90 \%$ (Figura 2).

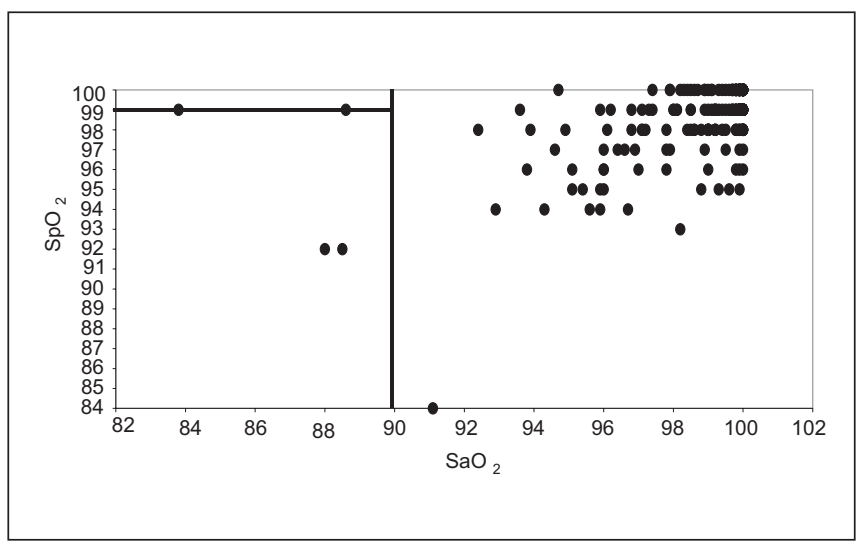

Figura 2 - Determinação Gráfica do Valor Mínimo da $\mathrm{SpO}_{2}$ para Evitar $\mathrm{SaO}_{2}$ menor que $90 \%$ 


\section{DISCUSSÃO}

O diagnóstico clínico de hipoxemia é dado pela presença de cianose. Entretanto, esta somente ocorre quando a $\mathrm{SaO}_{2}$ é menor que $75 \%$ e depende da concentração de hemoglobina reduzida, que deve ser superior a $5 \mathrm{~g} \cdot \mathrm{dl}^{-1}$. Assim, sua ausência não elimina a possibilidade de hipoxemia ${ }^{1}$. A saturação periférica da oxihemoglobina correlaciona-se fortemente com a saturação arterial da oxihemoglobina e, por esta razão é utilizada rotineiramente na monitorização peri-operatória e na terapia intensiva, permitindo o diagnóstico precoce de hipoxemia $^{3,5}$.

Os oxímetros de pulso aferem a diferença na absorção de dois comprimentos de onda luminosa durante sua passagem através de tecidos perfundidos, analisando a faixa de absorbância dos componentes contínuo e pulsátil do fluxo sangüíneo tecidual. As taxas de absorbância são relacionadas com medidas diretas de $\mathrm{SaO}_{2}$ obtidas em voluntários sadios por co-oximetria, resultando em um algoritmo de calibração, que é armazenado no microprocessador digital do oxímetro de pulso. Assim, é gerada uma estimativa da $\mathrm{SaO}_{2}{ }^{6}$.

Asaturação de oxigênio da oxihemoglobina no sangue arterial pode ser classificada em fracional e funcional. A primeira é medida espectrofotometricamente pela co-oximetria, sendo calculada pela relação $\left[\mathrm{HbO}_{2} /\left(\mathrm{HbO}_{2}+\mathrm{Hb}\right.\right.$ reduzida + metemoglobina + carboxi-hemoglobina) x 100]. A saturação de oxigênio funcional da oxihemoglobina é estimada pela oximetria de pulso e é calculada pela razão $\left[\mathrm{HbO}_{2} /\left(\mathrm{HbO}_{2}+\mathrm{Hb}\right.\right.$ reduzida) $\times 100]^{7}$. Desta maneira, a oximetria de pulso não diferencia a hemoglobina reduzida das demais formas de hemoglobina, podendo superestimar $\mathrm{SaO}_{2}$ na presença des$\operatorname{tas}^{7}$.

Artefatos na medida da $\mathrm{SpO}_{2}$ foram relacionados à diminuição da razão entre a qualidade do sinal e a interferência na captação. Assim, o uso de vasopressores, a baixa perfusão tissular e a colocação inadequada do sensor são responsáveis pela diminuição do sinal, enquanto o movimento e a luz ambiente aumentam a interferência ${ }^{8}$. A diminuição acentuada da resistência vascular sistêmica pode fazer com que a $\mathrm{SpO}_{2}$ subestime a $\mathrm{SaO}_{2}$. Em pacientes sépticos, o oxímetro de pulso é sensibilizado pelo fluxo venoso pulsátil, causado pela abertura de fístulas arterio-venosas cutâneas ${ }^{9}$. Embora a anemia aguda em pacientes não hipoxêmicos não afete significativamente a precisão da oximetria de pulso ${ }^{6}$, portadores de anemia falciforme, em vigência de crise falcêmica, apresentam valores de $\mathrm{SaO}_{2}$ superestimado pela $\mathrm{SpO}_{2}$, sem entretanto mascarar o diagnóstico de hipoxemia ${ }^{10,11}$. As dis-hemoglobinemias podem falsear as medidas da oximetria de pulso. Destas, a mais comum é a carboxi-hemoglobinemia, associada ao tabagismo crônico e à intoxicação pelo monóxido de carbono, em que a $\mathrm{SaO}_{2}$ é superestimada pela $\mathrm{SpO}_{2}{ }^{7}$. Durante metemoglobinemia grave, a $\mathrm{SpO}_{2}$ mantém-se constante, em torno de $85 \%$, independentemente do valor da $\mathrm{SaO}_{2}$. Metemoglobinemia pode resultar do uso de nitroprussiato de sódio, nitroglicerina, metoclopramida, sulfas, prilocaína e benzocaína ${ }^{12}$.
Fatores demográficos, como idade, sexo ou raça não influenciam a relação entre a $\mathrm{SpO}_{2}$ e $\mathrm{SaO}_{2}{ }^{13}$. Em negros, $\mathrm{SpO}_{2}$ abaixo de $95 \%$ reflete $\mathrm{SaO}_{2}$ menor que $90 \%$, enquanto, em brancos, o valor correspondente da $\mathrm{SpO}_{2}$ é $92 \%{ }^{6}$.

Os valores normais do gradiente $\mathrm{SpO}_{2}-\mathrm{SaO}_{2}$ variam entre menos e mais 3 a $5 \%{ }^{6}$.

Em anestesia e, especialmente em terapia intensiva, $\mathrm{a} \mathrm{SpO}_{2}$ é utilizada para indicar alterações do regime ventilatório. Assumindo valores normais do gradiente $\mathrm{SpO}_{2}-\mathrm{SaO}_{2}$, utilizam-se limites inferiores de $\mathrm{SpO}_{2}$ entre 92 e $94 \%$ para evitar $\mathrm{SaO}_{2}$ inferior a $90 \%$, que corresponde à hipoxemia leve ${ }^{3-6}$. Entretanto, em pacientes criticamente enfermos, sob ventilação mecânica, o limite inferior da $\mathrm{SpO}_{2}$ deveria ser de $96 \%$, para evitar hipoxemia durante alterações do regime ventilatório ${ }^{2}$.

Neste estudo, $95,28 \%$ dos pacientes apresentaram valores do gradiente $\mathrm{SpO}_{2}-\mathrm{SaO}_{2}$, dentro de dos limites de discordância, isto é, dois desvios padrão acima e abaixo da diferença média mas $4,72 \%$ apresentaram valores excessivamente altos. Nestes, caso a $\mathrm{SpO}_{2}$ mínima aceitável para afastar hipoxemia durante ajuste do regime ventilatório fosse estipulada entre 92 e $94 \%$, teriam resultado valores de $\mathrm{SaO}_{2}$ muito abaixo de $90 \%$ e os pacientes estariam hipoxêmicos após a intervenção.

Conclui-se que, embora a $\mathrm{SpO}_{2}$ estime a $\mathrm{SaO}_{2}$ com pequena discordância na grande maioria dos pacientes, somente valores iguais ou maiores que $99 \%$ afastam a possibilidade de $\mathrm{SaO}_{2}$ abaixo de $90 \%$ em pacientes cirúrgicos ou gravemente enfermos. Recomenda-se que alterações do regime ventilatório sejam feitas somente após confirmação gasométrica dos valores da $\mathrm{PaO}_{2}$ e da $\mathrm{SaO}_{2}$.

\section{$\mathrm{SpO}_{2}-\mathrm{SaO}_{2}$ Gap During Mechanical Ventilation in Anesthesia and Intensive Care}

Pablo Escovedo Helayel, M.D., Getúlio R. de Oliveira Filho, M.D., Lúcia Marcon, M.D., Flávio Hülse Pederneiras, M.D., Marcos Antônio Nicolodi, M.D., Sérgio GallufPederneiras, M.D.

\section{INTRODUCTION}

Peripheral oxy-hemoglobin saturation is a widely used estimate of its saturation in the arterial blood for monitoring patients under anesthesia and/or intensive care. During mechanical ventilation, $\mathrm{SpO}_{2}$ has been used to determine the adequacy of changes in ventilator settings, such as oxygen inspired fraction, positive tele-expiratory pressure values and weaning. $\mathrm{SpO}_{2}$ values traditionally associated to $\mathrm{SaO}_{2}$ higher than $90 \%$, that is, normoxemia, vary between $92 \%$ and $94 \%{ }^{1}$. Recently, however, the lack of hypoxemia has been only observed with $\mathrm{SpO}_{2}$ values equal to, or higher than $96 \%$. Such findings have obvious clinical implications. 
This study aimed to evaluated matching limits between $\mathrm{SpO}_{2}$ and $\mathrm{SaO}_{2}$ in anesthetized and critically ill patients during mechanical ventilation to establish minimum $\mathrm{SpO}_{2}$ values associated to more than $90 \%$ arterial oxy-hemoglobin saturation.

\section{METHODS}

After Hospital Governador Celso Ramos Medical Ethics Committee approval, participated in this prospective study 120 patients of both genders submitted to mechanical ventilation during general anesthesia or intensive care, with inspired oxygen fraction between $40 \%$ and $100 \%$, regardless of other ventilation parameters or of hemodynamic status. Patients in cardio-respiratory bypass, icteric, with nail polish or anemic (hemoglobin lower than $9 \mathrm{~g} \cdot \mathrm{ml}^{-1}$ ) were excluded from the study. Patients with $\mathrm{SaO}_{2}$ equal to, or lower than $75 \%$ were also excluded due to the possibility of venous contamination of the sample during arterial puncture. Arterial blood samples were collected by radial artery puncture or arterial line aspiration using an anaerobic technique and heparin-containing syringes. Samples were analyzed immediately after collection. During collection, $\mathrm{SpO}_{2}$ values were obtained with a pulse oximeter with reusable digital sensors, using the arterial pulse dichroic wave as the adequacy parameter.

$\mathrm{SpO}_{2}$ and $\mathrm{SaO}_{2}$ values were compared by Mann-Whitney $U$ test and their matching was evaluated by Bland-Altman test. To determine the lowest $\mathrm{SpO}_{2}$ value associated to an $\mathrm{SaO}_{2}$ equal to, or higher than $90 \%$ an $\mathrm{SaO}_{2}, \mathrm{SpO}_{2}$ chart was used where two lines were plotted. The vertical line defined $\mathrm{SaO}_{2}$ limit in $90 \%$ and the horizontal line crossed the ordinate axle at an $\mathrm{SpO}_{2}$ value above which no $\mathrm{SaO}_{2}$ values below $90 \%$ were observed.

\section{RESULTS}

Demographics data of 120 patients of whom 228 samples were analyzed are shown in table I. $\mathrm{SpO}_{2}$ values were significantly different from $\mathrm{SaO}_{2}$ values (Table II). Differences between $\mathrm{SpO}_{2}$ and $\mathrm{SaO}_{2}$ varied from $-7.10 \%$ to $15.20 \%$. Mean difference was $-0.20 \%$ with a standard deviation of $2.02 \%$. $\mathrm{SpO}_{2}-\mathrm{SaO}_{2}$ gap remained between plus or minus $4.04 \%$ (two standard deviations of the difference) in $95.28 \%$ of samples (Figure 1).

Table I - Demographics Data

\begin{tabular}{lc}
\hline Age (years) * & $52.94 \pm 16.56$ \\
Gender \# & \\
Masculino & 66 \\
Feminino & 54 \\
Weight $(\mathrm{kg}){ }^{*}$ & $71.60 \pm 15.97$ \\
Height $(\mathrm{m}){ }^{*}$ & $1.65 \pm 0.09$ \\
\hline
\end{tabular}

* Values expressed in Mean $\pm \mathrm{SD}$

\# Number of cases
Table II - Comparison between $\mathrm{SaO}_{2}$ and $\mathrm{SpO}_{2}$ values

\begin{tabular}{lc}
\hline Parameter & $\begin{array}{c}\text { Median [lower; upper quartile] } \\
\text { (Lowest - Highest) }\end{array}$ \\
\hline $\mathrm{SaO}_{2}$ & $99.8[98.5 ; 100]^{*}$ \\
& $83-100$ \\
$\mathrm{SpO}_{2}$ & $99[98 ; 100]$ \\
& $84-100$ \\
\hline
\end{tabular}

${ }^{*} \mathrm{p}<0.05$ as compared to $\mathrm{SpO}_{2}$

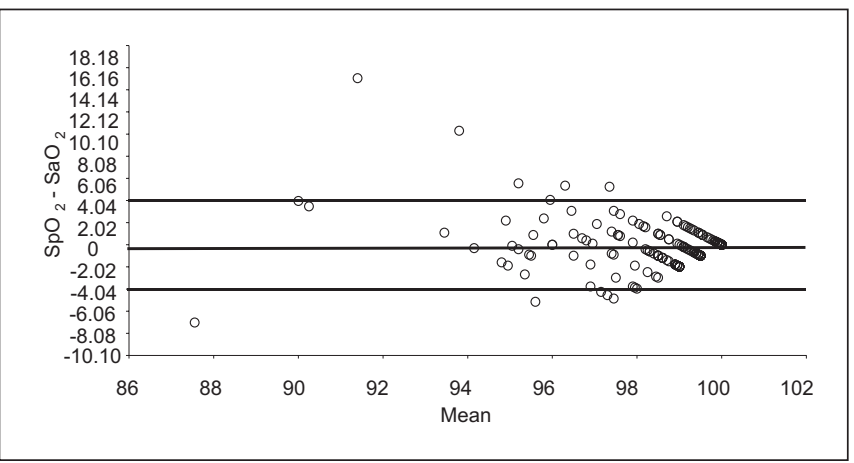

Figure 1 - Bland-Altman Diagram for $\mathrm{SpO}_{2}-\mathrm{SaO}_{2}$

Lowest $\mathrm{SpO}_{2}$ to assure an $\mathrm{SaO}_{2}$ higher than $90 \%$ has shown that only $\mathrm{SpO}_{2}$ values equal to, or higher than $99 \%$ were not associated to $\mathrm{SaO}_{2}$ values below $90 \%$ (Figure 2).

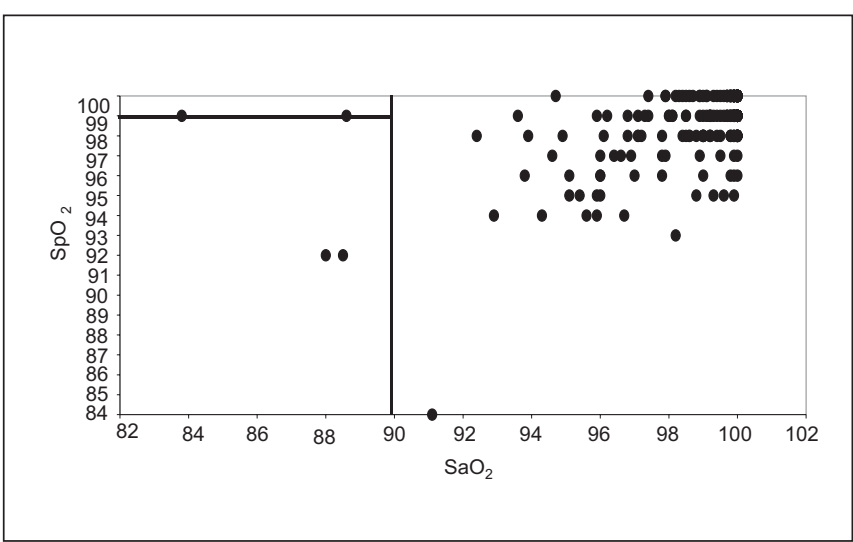

Figure 2 - Graphic Representation of Minimum $\mathrm{SpO}_{2}$ Value to Prevent $\mathrm{SaO}_{2}$ below $90 \%$

\section{DISCUSSION}

Hypoxemia is confirmed by the presence of cyanosis. However, it is only seen when $\mathrm{SaO}_{2}$ is lower than $75 \%$ and depends on reduced hemoglobin saturation, which must be higher than $5 \mathrm{~g} . \mathrm{dl}^{-1}$. So, its absence does not rule out the possibility of hypoxemia ${ }^{1}$. Peripheral oxy-hemoglobin saturation is strongly correlated to oxy-hemoglobin arterial saturation and, 
as such, it is routinely used in perioperative monitoring and in intensive care units for an early hypoxemia diagnosis ${ }^{3,5}$. Pulse oximetry checks the difference in absorption of two light wave lengths crossing perfused tissues, by analyzing the absorbance range of continuous and pulsatile components of tissue blood flow. Absorbance rates are related to $\mathrm{SaO}_{2}$ measurements obtained in healthy volunteers by co-oximetry, resulting in a calibration algorithm which is stored in the pulse oximeter's digital microprocessor and generates an $\mathrm{SaO}_{2}$ estimate ${ }^{6}$.

Arterial oxy-hemoglobin oxygen saturation may be classified as fractional and functional. The first is spectrophotometrically measured by co-oximetry and is calculated by the $\left[\mathrm{HbO}_{2} /\left(\mathrm{HbO}_{2}+\right.\right.$ reduced $\mathrm{Hb}+$ methemoglobin + carboxy-hemoglobin) $x$ 100] ratio. Functional oxy-hemoglobin oxygen saturation is estimated by pulse oximetry and is calculated by the $\left[\mathrm{HbO}_{2} /\left(\mathrm{HbO}_{2}+\right.\right.$ reduced $\left.\left.\mathrm{Hb}\right) \times 100\right]$ ratio ${ }^{7}$. This way, pulse oximetry does not distinguish reduced hemoglobin from other forms of hemoglobin and may overestimate $\mathrm{SaO}_{2}{ }^{7}$. $\mathrm{SpO}_{2}$ measurements artifacts were related to a decrease in the ratio between signal quality and capturing interference. So, the use of vasopressants, the low tissue perfusion and the inadequate placement of the sensor are responsible for signal decrease, while movement and room light increase the interference ${ }^{8}$. The marked decrease in systemic vascular resistance may lead $\mathrm{SpO}_{2}$ to underestimate $\mathrm{SaO}_{2}$. In septic patients, pulse oximeter is sensitized by the pulsatile venous flow caused by the opening of skin arterial-venous fistulas ${ }^{9}$. Although acute anemia in non hypoxemic patients does not significantly affect pulse oximetry precision ${ }^{6}$, sickle cell disease patients during crisis show $\mathrm{SaO}_{2}$ values underestimated by $\mathrm{SpO}_{2}$, however without hiding the diagnosis of hypoxemia ${ }^{10,11}$. Dyshemoglobulinemias may affect pulse oximetry measurements. From those, the most common is carboxy-hemoglobulinemia associated to chronic smoking and $\mathrm{CO}_{2}$ poisoning, where $\mathrm{SaO}_{2}$ is overestimated by $\mathrm{SpO}_{2}$. During severe methemoglobinemia, $\mathrm{SpO}_{2}$ is constant around $85 \%$, regardless of $\mathrm{SaO}_{2}$. Methemoglobulinemia may result from the use of sodium nitroprusside, nitroglycerin, metoclopramide, sulfas, prilocaine and benzocaine ${ }^{12}$.

Demographics, such as age, gender or race do not influence $\mathrm{SpO}_{2}-\mathrm{SaO}_{2}$ ratio ${ }^{13}$. In blacks, $\mathrm{SpO}_{2}$ below $95 \%$ reflects $\mathrm{SaO}_{2}$ below $90 \%$, while in Caucasians this same $\mathrm{SpO}_{2}$ value is $92 \%{ }^{6}$.

Normal $\mathrm{SpO}_{2}-\mathrm{SaO}_{2}$ gap values vary between plus or minus $3 \%$ to $5 \%{ }^{6}$.

In anesthesia, and especially in intensive care, $\mathrm{SpO}_{2}$ is used to identify changes in ventilator settings. Assuming normal $\mathrm{SpO}_{2}-\mathrm{SaO}_{2}$ gap values, lower $\mathrm{SpO}_{2}$ values between $92 \%$ and $94 \%$ are used to prevent $\mathrm{SaO}_{2}$ going below $90 \%$, which corresponds to mild hypoxemia ${ }^{3-6}$. However, in critically ill patients under mechanical ventilation, lower $\mathrm{SpO}_{2}$ limit should be $96 \%$ to avoid hypoxemia during changes in ventilator settings ${ }^{2}$.

In our study, $95.28 \%$ of patients showed $\mathrm{SpO}_{2}-\mathrm{SaO}_{2}$ gap values within mismatching limits, that is, two standard deviations above and below the mean difference, but $4.72 \%$ showed excessively high values. In such cases, if the lowest acceptable $\mathrm{SpO}_{2}$ to rule out hypoxemia during ventilator settings adjustment was established between $92 \%$ and $94 \%$, $\mathrm{SaO}_{2}$ values would be well below $90 \%$ and patients would be hypoxemic after the intervention.

We concluded that, although $\mathrm{SpO}_{2}$ estimating $\mathrm{SaO}_{2}$ with a minor mismatch in most cases, only values equal to, or higher than $99 \%$ rule out the possibility of $\mathrm{SaO}_{2}$ below $90 \%$ in surgical or critically ill patients. It is recommended that adjustments in ventilator settings should only be done after confirmation of $\mathrm{PaO}_{2}$ and $\mathrm{SaO}_{2}$.

\section{REFERÊNCIAS - REFERENCES}

01. Powell, JF, Menon DK, Jones JG - The effects of hypoxemia and recommendations for postoperative oxygen therapy. Anaesthesia, 1996;51:769-772.

02. Seguin P, Le Rouzo A, Tanguy M et al - Evidence for the need of bedside accuracy of pulse oximetry in an intensive care unit. Crit Care Med, 2000;28:703-706.

03. Moller JT, Johannessen NW, Espersen K et al - Randomized evaluation of pulse oximetry in 20,802 patients: I. Perioperative events and postoperative complications. Anesthesiology, 1993; 78:445-453.

04. Jensen L.A, Onyskiw JE, Prasad NG - Meta-analysis of arterial oxygen saturation monitoring by pulse oximetry in adults. Heart Lung, 1998;27:387-408.

05 . Smith DC - Pulse oximetry in the recovery room. Anaesthesia, 1989;44:345-348.

06. Jubran A - Advances in respiratory monitoring during mechanical ventilation. Chest, 1999;116:1416-1425.

07 . Hampson $\mathrm{N}$ - Pulse oximetry in severe carbon monoxide poisoning. Chest, 1998;114:1036-1041.

08. Severinghaus JW, Spellman MJ - Pulse oximeter failure thresholds in hypotension and vasoconstriction. Anesthesiology, 1990;73:532-537.

09. Secker C, Spiers P - Accuracy of pulse oximetry in patients with low systemic vascular resistance. Anaesthesia, 1997;52: 127-130.

10. Kress JP, Pohlman AS, Hall JB - Determination of hemoglobin saturation in patients with acute sickle chest syndrome: a comparison of arterial blood gases and pulse oximetry. Chest, 1999;115:1316-1320.

11. Ortiz FO, Aldrich TK, Nagel RI et al - Accuracy of pulse oximetry in sickle cell disease. Am J Respir Crit Care Med, 1999;159: 447-451.

12. Barker SJ, Tremper KK, Hyatt J et al - Effects of methemoglobin on pulse oximetry and mixed venous oximetry. Anesthesiology, 1989;70:112-117.

13. Saber W, McCarthy K, Schilz R - Limitations of pulse oximetry. Chest, 2000;118:184S.

\section{RESUMEN}

Helayel PE, Oliveira Filho GR, Marcon L, Pederneiras FH, Nicolodi MA, Pederneiras SG - Gradiente $\mathrm{SpO}_{2}-\mathrm{SaO}_{2}$ Durante Ventilación Mecánica en Anestesia y Terapia Intensiva

Justificativas y Objetivos - La saturación periférica de la oxihemoglobina $\left(\mathrm{SpO}_{2}\right)$ es frecuentemente utilizada para guiar alteraciones del régimen ventilatorio. Valores de $\mathrm{SpO}_{2}$ iguales o superiores a $96 \%$ son necesarios para garantizar saturación 
arterial de la oxihemoglobina $\left(\mathrm{SaO}_{2}\right)$ superiores a $90 \%$, en pacientes de terapia intensiva. Este estudio tuvo por objetivo determinar concordancia entre valores de $\mathrm{SpO}_{2}$ y $\mathrm{SaO}_{2}$ y delimitar la menor $\mathrm{SpO}_{2}$ asociada con valores de $\mathrm{SaO}_{2}$ iguales o superiores a $90 \%$.

Método - Fueron incluidos prospectivamente 120 pacientes adultos, de ambos sexos, sometidos a anestesia general con ventilación mecánica o en tratamiento intensivo. Muestras de sangre arterial fueron colectadas por punción arterial o por aspiración de línea arterial, utilizando técnica anaeróbia, en jeringas heparinizadas. Las muestras fueron analizadas inmediatamente después de la colecta. $\mathrm{La} \mathrm{SpO} \mathrm{S}_{2}$ fue medida por oxímetro de pulso durante la colecta, en el indicador de la mano contra-lateral, utilizando la onda dicrótica de pulso arterial como parámetro de adecuación.
Resultados - Fueron analizadas 228 muestras. La diferencia entre los valores de $\mathrm{SpO}_{2}$ y $\mathrm{SaO}_{2}$ varió entre $-7,10 \%$ y 15,20\%, siendo la diferencia media igual a $-0,20 \% \pm 2,02 \%$. SpO $-\mathrm{SaO}_{2}$ mayores que $4,04 \%$ (dos desvíos padrones de la diferencia) ocurrieron en $4,72 \%$ de las muestras. La determinación gráfica de la $\mathrm{SpO}_{2}$, mínima para garantizar la $\mathrm{SaO}_{2}$ arriba de $90 \%$, mostró que solamente valores de $\mathrm{SpO}_{2}$ iguales o superiores a $99 \%$ no se asociaron con la ocurrencia de ningún valor de $\mathrm{SaO}_{2}$ inferior a $90 \%$.

Conclusiones - Aun cuando la diferencia entre los valores de $\mathrm{SpO}_{2}$ y $\mathrm{SaO}_{2}$ se hallan localizados entre más y menos $5 \%$ en $97 \%$ de los pares analizados, solamente valores arriba de $99 \%$ excluyeron pacientes con $\mathrm{SaO}_{2}$ menor que $90 \%$. 